\title{
NITRIC OXIDE AND SYSTEMIC SCLEROSIS
}

\author{
Lubica Cibičková1, Tomáš Soukup ${ }^{1}$, Norbert Cibiček ${ }^{2}$, Jaroslav Chládek ${ }^{3}$
}

Charles University in Prague, Faculty of Medicine in Hradec Králové and University Hospital Hradec Králové, Czech Republic: $2^{\text {nd }}$ Department of Medicine $^{1}$; Institute for Clinical Biochemistry and Diagnostics ${ }^{2}$; Department of Pharmacology 3

Key words: Systemic sclerosis; Nitric oxide

Vascular endothelial dysfunction is a central event in the pathogenesis of a variety of human diseases, including systemic sclerosis. Systemic sclerosis ( $\mathrm{SSc}$ ) is a complex connective tissue disease that involves vascular dysfunction, immunological activation and fibrosis. In its elusive etiopathogenesis, nitric oxide (NO) is supposed to be involved. NO accounts for the activities originally ascribed to "endothelium-derived relaxing factor." It is produced by all mammalian cells and provides pleiotropic properties. NO is produced by three nitric oxide synthase (NOS) isoforms: two are constitutive (endothelial eNOS and neuronal nNOS) and one is inducible (iNOS). Constitutive forms are $\mathrm{Ca} 2+-$ and calmodulin-dependent enzymes and release, femtomolar or picomolar concentrations of NO in reaction to receptor stimulation. Inducible NOS is regulated at a pretranslational level and can be induced by several proinflammatory cytokines, such as tumor necrosis factor-alpha (TNF- $\alpha$ ), interferon-gamma (IFN- $\gamma$ ), and interleukin-1 (IL-1). iNOS releases large quantities (nanomolar concentrations) of NO several hours after exposure. Constitutive forms are active under physiological conditions, whilst the latter participates in tissue pathology (e.g. during inflammatory autoimmune disease as a contributor to tissue destruction). Hence, the molecule may act both as a regulatory mediator and as a potent cytotoxic agent (Kolb, 1998). This „nitric oxide paradox“ has been found to exist also in SSc, where NO may play a positive (vasodilating) as well as negative (cytotoxic, causing tissue dysfunction and injury) role (Cerinic, 2002). However, the opposing effects of NO in SSc seem not to be simply explainable. This is supported by the fact, that literature is inconsistent even regarding the nature of change (increase or decrease) in NO level in SSc patients compared to healthy subjects. Furthermore, the controversial issue gains in complexity as the levels of NO are shown to vary depending on the location, analyzed material, disease activity and progression:

1) Biopsies of microvessels

In biopsies from patients with SSc, superficial microvessels showed a peak of eNOS expression (detected by im- munohistochemical staining) in indurated skin which fell as the changes of the skin increased. By contrast, iNOS staining increased with the grade of skin damage (Cotton, 1999). Analogous to these results Romero described reduced expression of eNOS mRNA by dermal microvascular endothelial cells from patients with SSc (Romero, 2000). Described switch from eNOS to iNOS as the disease progresses may demonstrate both roles of NO - first positive and than cytotoxic.

\section{2) Serum}

Yamamoto observed significantly higher levels of serum NO metabolite nitrite in patients with SSc (Yamamoto, 1998), a finding later confirmed by other researchers (Sud, 2000; Takagi, 2003). Sud showed an association between worsening of lung functions (assessed by spirometry) and a trend towards higher serum NO levels (resp. nitrite and citrulline), even though no difference in NO levels among disease subgroups could be found (corrected for the extent of disease, disease duration, age and systemic involvement) (Sud, 2000). Nevertheless, some clinical features of SSc (the extent of skin fibrosis, short disease duration and fibrosing alveolitis) seem to give positive correlations with serum levels of NO metabolites (Takagi, 2003).

3) Peripheral blood mononuclear cells

Although not reaching statistical significance, the amounts of spontaneously released NO from blood mononuclear cells of patients with SSc exceed those of control subjects' (Yamamoto, 1998). In contrast, a more recent study showed that the concentrations of NO metabolites are decreased and the induction of iNOS is delayed in cultured peripheral blood mononuclear cells in patients with SSc (Allanore, 2001).

\section{4) Exhaled air}

The pulmonary production of NO may be elevated (Fajac, 1998, Kharitonov, 1997, Rolla, 2000) as lung macrophages express higher amounts of iNOS (Failli, 2002) in SSc patients compared to healthy controls. On the other hand, some found NO levels to be decreased in patients suffering from SSc with pulmonary hypertension (Kharitonov, 
Tab. 1.

\begin{tabular}{|l|c|c|c|c|}
\hline Author, year & Nitric oxide & No. of SSc patients & Method & Analysed material \\
\hline Rolla, 2000 & $\uparrow(\downarrow$ in PAH $)$ & $47(16$ with PAH $)$ & chemiluminiscence & Exhaled air \\
\hline Kharitonov, 1997 & $\uparrow(\downarrow$ in PAH $)$ & $23(6$ with PAH $)$ & chemiluminiscence & Exhaled air \\
\hline Fajac, 1998 & $\uparrow$ & 14 & chemiluminiscence & Exhaled air \\
\hline Failli, 2002 & $\uparrow$ & 32 & colorimetry & Lung macrophages \\
\hline Yamamoto, 1998a & $\uparrow$ & 14 & colorimetry & PBMC \\
\hline Allanore, 2001 & $\downarrow$ & 18 & spectrophotometry & PBMC \\
\hline Yamamoto, 1998b & $\uparrow$ & $?$ & colorimetry & Serum \\
\hline Takagi, 2003 & $\uparrow$ & 45 & colorimetry & Serum \\
\hline Sud, 2000 & $\uparrow$ & 34 & colorimetry & Serum \\
\hline
\end{tabular}

$\mathrm{PAH}=$ pulmonary arterial hypertension; $\mathrm{PBMC}=$ peripheral blood mononuclear cells

1997; Rolla, 2000). Moreover, an inverse correlation between pulmonary hypertension and exhaled NO seems to exist (Rolla, 2000). The impairment of NO production in pulmonary arterial hypertension due to SSc may result from endothelial dysfunction characteristic for pulmonary arterial hypertension.

Above cited papers refer in good accords to elevated production of NO in serum and exhaled air. These changes reflect the situation in the whole body, where NO may act cytotoxic on vessels and cause their inflammation and damage. However, there is only little evidence about the situation directly in the skin and subcutis, which are affected by immunopathological process in the course of the disease.

Studying the relationship between NO and SSc brings a few problems. The results may vary depending on the used analytical methods, analyzed material (Table 1), patients' diet etc. In addition, the amount of NO produced may also differ with progression and activity of the disease, a fact that only a few authors took into account. For example, Cotton demonstrated a shift from eNOS to iNOS with progression of SSc (Cotton, 1999) and Yamamoto pointed out a correlation of total circulating NO with markers of endothelial damage and disease activity (Yamamoto, 1998). Moreover, the numbers of SSc patients included in the studies are rather low (Table 1).

Thus, further and larger studies are necessary to clarify the role NO plays in the development of SSc. The question of treating SSc patients with NO supplementation/inhibition at a particular stage and activity of the disease remains to be fully answered. The measurement of total NO in serum is unlikely to be representative for local contribution of this mediator to the pathogenesis of SSc. Therefore, a serial determination of NO production in situ respecting the pro- gression of the disease is suggested as an optimal alternative.

This report has been supported by research project $M Z O$ 00179906.

\section{References}

1. Allanore Y, Borderie D, Hilliquin P et al. Low levels of nitric oxide (NO) in systemic sclerosis: inducible NO synthase production is decreased in cultured peripheral blood monocyte/macrophage cells. Rheumatology 2001;40:1089-96.

2. Cerinic MM, Kahaleh MB. Beauty and the Beast. The nitric oxide paradox in systemic sclerosis. Rheumatology 2002;41;843-7.

3. Cotton AS, Herrick AL, Jayson MIV, Freemont AJ. Endothelial expression of nitric oxide synthases and nitrotyrosine in systemic sclerosis skin. J Pathol 189:273-279 (1999)

4. Failli P, Palmieri L, D'Alfonso C et al. Effect of N-acetyl-L-cysteine on peroxynitrite and superoxide anion production of lung alveolar macrophages in systemic sclerosis. Nitric oxide 2002;7:277-82.

5. Fajac I, Kahan A, Menkes CJ, Dessanges JF, Dall'Ava-Santucci J, Dinh-Xuan AT Increased nitric oxide in exhaled air in patients with systemic sclerosis. Clin Exp Rheumatol. 1998;16(5):547-52

6. Kharitonov SA, Cailes JB, Black CM, du Bois RM, Barnes PJ. Decreased nitric oxide in the exhaled air of patients with systemic sclerosis with pulmonary hypertension. Thorax. 1997:52(12):1051-5

7. Kolb H, Kolb-Bachofen V. Nitric oxide in autoimmune disease: cytotoxic or regulatory mediator? Immunology today 1998;19(12):556-61.

8. Rolla G, Colagrande P, Scappaticci E et al. Exhaled nitric oxide in systemic sclerosis: relationships with lung involvement and pulmonary hypertension. J Rheumatol. $2000 \mathrm{Jul} ; 27(7): 1693-8$.

9. Romero LI, Zhang DN, Cooke JP et al. Differential expression of nitric oxide by dermal microvascular endothelial cells from patients with scleroderma. Vasc Med. 2000;5(3):147-58

10. Sud A, Khullar M, Wanchu A, Bannery P. Increased nitric oxide production in patiens with systemic sclerosis. Nitric oxide: Biology and Chemistry 2000;4(6): $615-9$.

11. Takagi K, Kawaguchi Y, Hara M, Sugiura T, Harigai M, Kamatani N. Serum nitric oxide (NO) levels in systemis sclerosis patients: correlation between NO levels and clinical features. Clin Exp Immunol 2003;134:538-544.

12. Yamamoto T, Sawada Y, Katayama I, Nishioka K. Increased production of nitric oxide stimulated by interlaukin- $1 \beta$ in peripheral blood mononuclear cells in patients with systemic sclerosis. British Journal of Rheumatology 1998;37:1123-5.

13. Yamamoto T, Katayama I, Nishioka K. Nitric oxide production and inducible nitric oxide synthase experssion in systemic sclerosis. J Rheumatol. 1998 Feb; 25(2)314-7.

\section{Corresponding author:}

MUDr. Lubica Cibičková, University Hospital Hradec Králové, $2^{\text {nd }}$ Department of Medicine,

Sokolská 581, 50005 Hradec Králové, Czech Republic, e-mail: cibickova@seznam.cz 